\title{
Wetting behaviors of water droplet on rough metal substrates
}

\author{
Jing $\mathrm{Xu}^{1}{ }^{1} \mathrm{a}^{*}$, Bin $\mathrm{Li}^{1}$, Jiadi Lian ${ }^{2}$, Jing $\mathrm{Ni}^{1}$, Jing Xiao ${ }^{1}$ \\ ${ }^{1}$ Department of Mechanical Engineering, Hangzhou Dianzi University, Hangzhou, 310018, China. \\ ${ }^{2}$ Department of Mechanical Engineering, Zhejiang SCI-TECH University, Hangzhou, 310018, \\ China. \\ ${ }^{a}$ xujing@hdu.edu.cn
}

Keywords: Wetting behavior, Roughness, Metal, Static contact angle

\begin{abstract}
In this paper, an experimental investigation of wetting behaviors of water droplet on rough metal substrates is presented, in order to study the influence of rough structures on contact angles of metal substrates. The static contact angles and contact angles evolution over time are measured with a video contact angle analyzer. The results show that the hydrophobic property increases with increase of surface roughness. The contact angle increases from $76.8^{\circ}$ to $86.7^{\circ}$ with increasing mean roughness from $1.435 \mu \mathrm{m}$ to $5.591 \mu \mathrm{m}$. The surface with large aspect ratio displays larger contact angle than surface with low aspect ratio. The apparent decrease of the variation of contact angle as a linear variation with the time changing, the droplet spreads on flat substrates with low roughness slowly. Compare with Cassie state, Wenzel wetting state is more sensitive to the change of surface roughness. It may be expected to use the reasonable lapping process and parameters to control the static contact angle of water droplets on the metal surfaces.
\end{abstract}

\section{Introduction}

The surface rough structures of mechanical equipments is closely related to its performance, the purpose of researching surface roughness is controlling and improving the product quality. Specially, with the development of the precision of modern machinery manufacturing technology, the influence of surface roughness on mechanical performance becomes the leading-edge research, and one of performance influence factors is wetting behaviors, which become a research hotspot in the fields of fluid interface. Metal as an important engineering material play an irreplaceable role in industrial application. It is well-known that metal with high surface energy is typical inherently hydrophilic material. The influence of surface roughness on wetting behaviors of metal substrates must be known in advance are expected so as to improve the wear-resisting performance by controlling droplets shape on the surface, which largely depends on its spreading property [1,2], the dynamic wetting [3], but not just only on the static wetting property [4].

Several recent experiments have been reported dealing with this topography effect on surface wettability [5-8]. Andrew et al. [9] analyzed and compared the properties of a growing frost layer for surfaces of different wettability, to determine the effect that the surface energy has on the frost mass, thickness, and density. Du et al. [10] studied surface wettability modification of polyimide film by UV laser direct texturing (UVLDT). It was found that in the same modification atmosphere, surface microstructure and roughness played a major role in changing the wetting behavior of polyimide film with varied laser fluence. Wu et al. [11] carried out comparative studies of surface wettability of ground $\mathrm{C} / \mathrm{SiC}$ surface and laser-ablated $\mathrm{C} / \mathrm{SiC}$ surface through apparent contact angle (APCA) measurements. The results showed that water droplets easily reached stable state on ground $\mathrm{C} / \mathrm{SiC}$ surface. Atsuhide et al. [12] presented an experimental study of the influence of wall surface wettability on the behavior of hydrogen microbubbles rising along a nearly vertical wall. The microbubble behavior observed along three different kinds of wall surfaces (hydrophobic, hydrophilic, and super-hydrophilic) was characterized by the microbubble-wall attachment, bubble size distribution, bubble coalescence, and microbubble layer formation. All these works show that roughness surface may achieve different wetting surface, which provide a potential way to control 
the static contact angle on the metal surfaces.

In this work, we investigate the wetting behaviors of water droplet on rough metal substrates, in order to study the influence of rough structures on contact angles of metal substrates. AISI 304L stainless steel samples were polished to investigate the impact of roughness on wettability property on metal substrates, Generally, preparation of rough surface is essential processes, and the number of mean roughness is varied. The static contact angles and contact angles evolution over time are measured with a video contact angle analyzer.

\section{Experimental Section}

AISI 316L stainless steel samples are mechanically polished to obtain mirror polished surfaces. Samples are cleaned after treatments with a 15 min ultrasonic bath in acetone. The clean, smooth and thin metal substance is polished by lapping machine with different sizes of abrasive particle or sandpaper for $15 \mathrm{~min}$, and follow by rinsing with acetone for $3 \mathrm{~min}$. Roughness was measured by the digital microscope with large depth-of-filed imaging. Fig.1 shows the mean roughness of those samples (sample I, II, III, IV, V) are $1.435 \mu \mathrm{m}, 3.006 \mu \mathrm{m}, 4.448 \mu \mathrm{m}, 5.591 \mu \mathrm{m}$ and $5.721 \mu \mathrm{m}$, respectively. The difference between sample $I V$ and sample $V$ is the surface feature of aspect ratio. The sample $\mathrm{V}$ is the only sample which is rubbed with fine sandpaper, then polish with lapping machine, the above lapping process directly result in the multiple scales surface roughness with high aspect ratio.

The static contact angles and contact angles evolution over time are measured with a video contact angle analyzer.

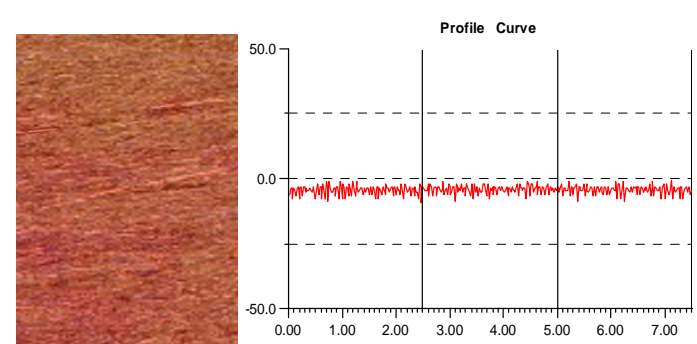

(a) sample I $(1.435 \mu \mathrm{m})$

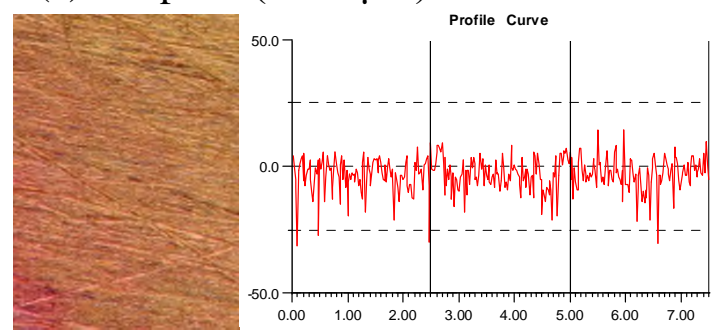

(c) sample III $(4.448 \mu \mathrm{m})$

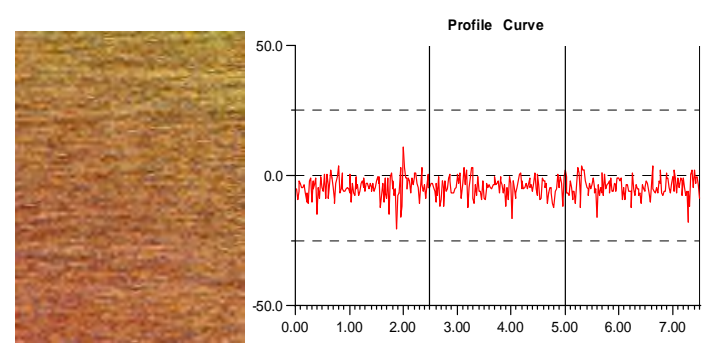

(b) sample II $(3.006 \mu \mathrm{m})$
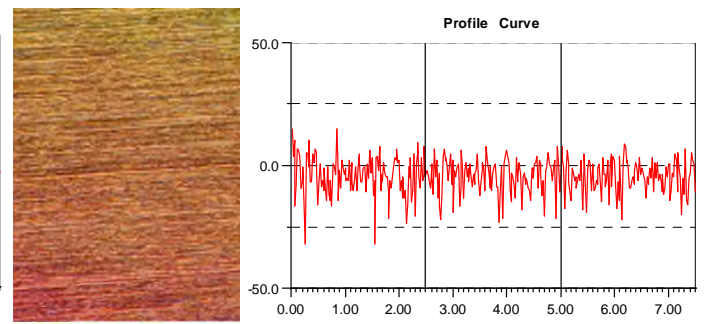

(d) sample IV $(5.591 \mu \mathrm{m})$

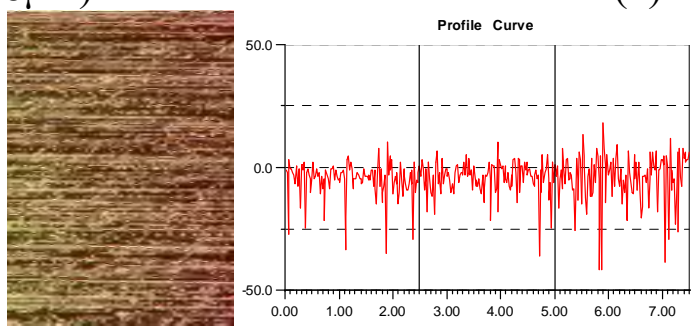

(e) sample V $(5.721 \mu \mathrm{m})$

Fig. 1 Optical images and profiles of rough surface (a) mean roughness $\mathrm{Ra}=1.435 \mu \mathrm{m}$;

(b) $\mathrm{Ra}=1.435 \mu \mathrm{m}$; (c) $\mathrm{Ra}=4.448 \mu \mathrm{m}$; (d) $\mathrm{Ra}=5.591 \mu \mathrm{m}$; (e) $\mathrm{Ra}=5.721 \mu \mathrm{m}$. 


\section{Results and Discussion}

Fig. 2 shows that the spreading dynamics of a $4 \mu 1$ droplet on different roughness surfaces. When time $\mathrm{t}=0 \mathrm{~s}$, the different roughness substrates correspond to different static contact angle, the hydrophobic property increases with increase of surface roughness. The contact angle increases from $76.8^{\circ}$ to $86.7^{\circ}$ with increasing mean roughness from $1.435 \mu \mathrm{m}$ to $5.591 \mu \mathrm{m}$. Comparing to sample IV with low aspect ratio, the contact angle of sample $\mathrm{V}$ with large aspect ratio reaches $92.9^{\circ}$. It indicates that the surface feature of aspect ratio is so important to static contact angle, the water droplet is prevent to penetrate into rough surface structure with large aspect ratio. The specific hydrophobic behaviors (the contact angles were bigger than $90^{\circ}$ ) with high roughness are due to two kinds of reasons, the first is the pinning of gas-fluid interface, and the second is the air pockets between the solid and fluid. The rough structures increase the solid surface hydrophobic with the decrease of the solid-liquid contact area. It may be expected to use the reasonable lapping process and parameters to control the static contact angle of water droplets on the metal surfaces.

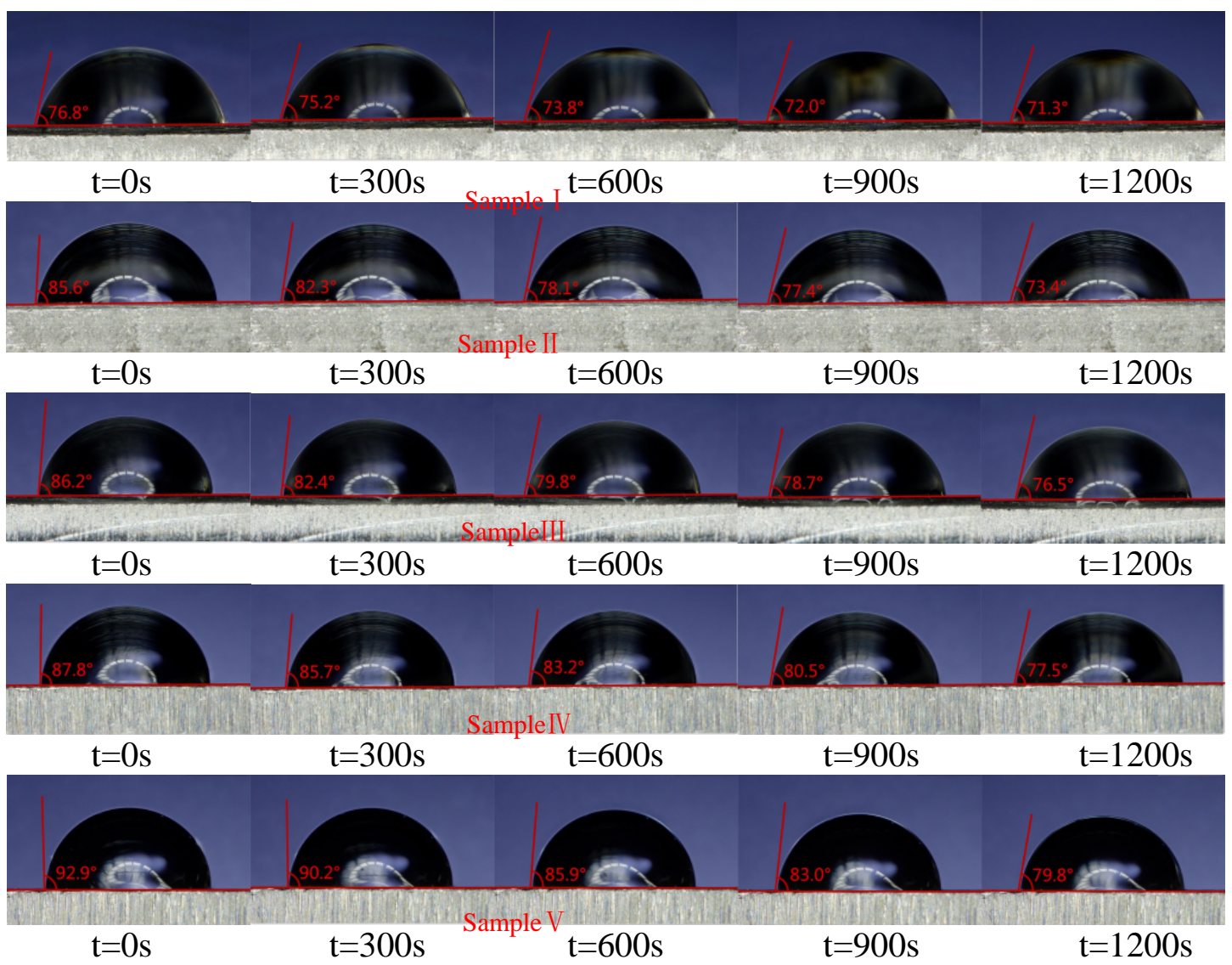

Fig. 2 Spreading dynamics of a $4 \mu 1$ droplet on different roughness surfaces (the magnification factor is 100 times). 


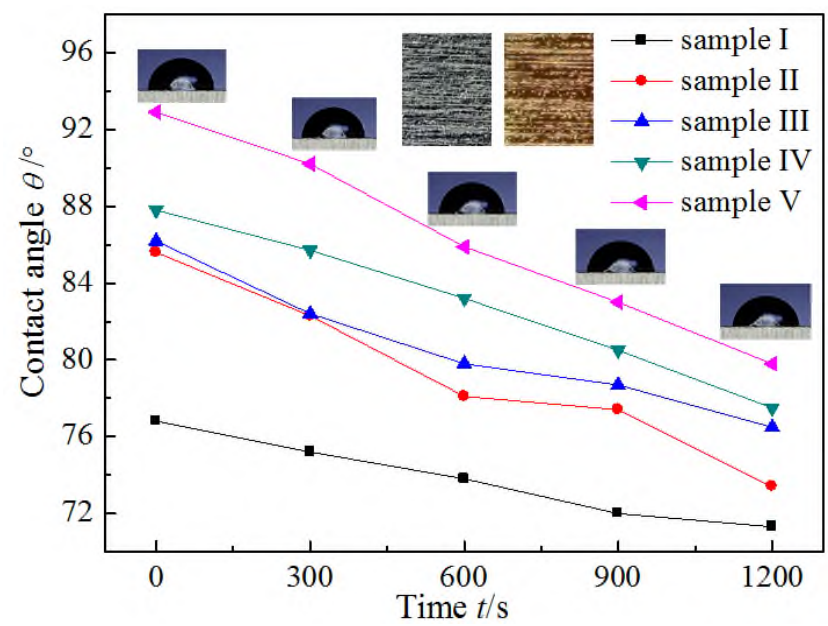

Fig. 3 Evolution of contact angle over time on different roughness surfaces.

Figs. 2 and 3 show that evolution of contact angle over time on different roughness surfaces. The apparent decrease of the variation of contact angle as a linear variation with the time changing, the velocity of droplets spread are depend on the state of wetting transitions. The droplet spread on flat substrates with low roughness slowly, the pace of expansion is slow on flat substrates than that on the rough surfaces. When time $t=0 \mathrm{~s}$, the situation is somewhat complicated by the presence of multiple local free energy minima leading to a composite interface, it is so-called metastable Cassie state (Fig. 4), which is caused by microscopic pockets of air remaining trapped below the liquid drople. Then the droplet spreads over time, the contact angle decreases progressively, the state change into almost completely Wenzel state but cannot reach the Wenzel perfect wetting state. Wenzel state is encountered only when the liquid drop has a complete contact with the rough surface [18]. Indeed, little air bubbles still get trapped at the interface. Compare with Cassie state, Wenzel state is more sensitive to the change of surface roughness.

Also, due to greater amount of spreading on the rough surfaces, the contact area is more, which gives more area for droplet seeped into a certain depth of rough surface. Accurate quantitative measurement of distribution morphology is irrespective and was not attempted in this work, the deeper depth of rough surface, the more gas expelled out of the gap, the more contact angle decease. The specific process between Wenzel-Cassie wetting state transition on rough patterned hydrophilic or hydrophobic material in detail needs further scrutiny.
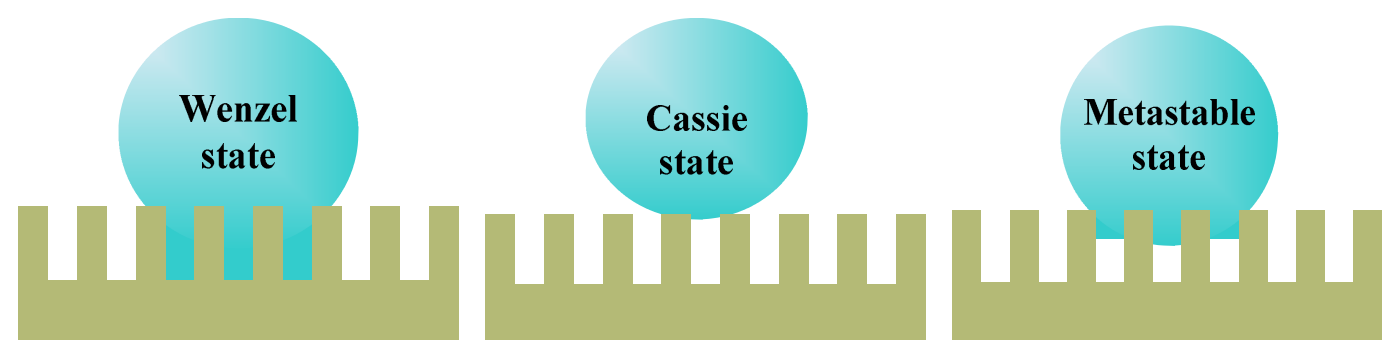

Fig. 4 Schematic illustrations of a droplet on rough surface, behavior of the drop in the case of Wenzel model, Cassie mode and Metastable model

\section{Conclusions}

In this paper, the rough structure drastically affects wetting behaviors of water droplet on rough metal substrate, the hydrophobic property increased with increase of surface roughness. The hydrophobic behaviors with high roughness are due to two reasons, the first is the pinning of gas-fluid interface, and the second is the air pockets between the solid and fluid. The surface with large aspect ratio displays larger contact angle than surface with low aspect ratio. It may be expected to use the reasonable lapping process and parameters to control the static contact angle of 
water droplets on the metal surfaces. The apparent decrease of the variation of contact angle as a linear variation with the time changing, the droplet spread on flat substrates with low roughness slowly, and it depends on the state of wetting transitions. Compare with Cassie state, Wenzel wetting state is more sensitive to the change of surface roughness. It may be expected to use the reasonable lapping process and parameters to control the static contact angle of water droplets on the metal surfaces.

\section{Acknowledgements}

The research is financially supported by the National Nature Science Foundation of China (No. 51505112, 51375129), the Nature Science Foundation of Zhejiang Province, China (No. LQ15E050010).

\section{References}

[1] D. Bonn, J. Eggers, J. Indekeu, et al. Wetting and spreading. Rev. Mod. Phys, 81(2) (2009) 739-804.

[2] H. Y. Erbil. ChemInform Abstract: evaporation of pure liquid sessile and spherical suspended drops: a review. ChemInform, 43(16) (2012) 67-86.

[3] S. K. Singh, S. Khanderkar, D. Pratap, et al. Wetting dynamics and evaporation of sessile droplets on nano-porous alumina surfaces. Colloid. Surf. A: Physicochem. Eng. Aspect. 432 (2013) 71-81.

[4] P. Bizi-Bandoki, S. Benayoun, S. Valette, et al. Modifications of roughness and wettability properties of metals induced by femtosecond laser treatment. Appl. Surf. Sci. 257(12) (2011) 5213-5218.

[5] Z. Yoshimitsu, A. Nakajima, T. Watanabe, et al. Effects of surface structure on the hydrophobicity and sliding behavior of water droplets. Langmuir, 18 (2002) 5818-5822.

[6] E. Stratakis, A. Ranella, C. Fotakis. Biomimetic micro/nanostructured functional surfaces for microfluidic and tissue engineering applications. Biomicrofluid. 5(1) (2011) 13411.

[7] C. H. Choi, C. J. Kim. Droplet evaporation of pure water and protein solution on nanostructured superhydrophobic surfaces of varying heights. Langmuir, 25(13) (2009) 7561-7567.

[8] R. N. Wenzel. Resistance of solid surfaces to wetting by water. Ind. Eng. Chem. 28 (1936) 988-994.

[9] A. D. Sommers, N. L. Truster, A. C. Napora, A. C. Riechman, E. J. Caraballo. Densification of frost on hydrophilic and hydrophobic substrates - Examining the effect of surface wettability. Exp. Therm. Fluid. Sci. 385(1) (1936) 391-399.

[10] Q. F. Du, J. G. Liu, L. B. Guo, M. Lv, X. Y. Zeng. Tailoring the surface wettability of polyimide by UV laser direct texturing in different gas atmospheres. Mater. Amp. Des. 75 (2016) 25-34.

[11] M. L. Wu, C. Z. Ren, H. Z. Xu. On the wettability diversity of C/SiC surface: Comparison of the ground $\mathrm{C} / \mathrm{SiC}$ surface and ablated $\mathrm{C} / \mathrm{SiC}$ surface from three aspects. Appl. Surf. Sci. 80 (2016) 126-138.

[12] A. Kitagawa, P. Denissenko, Y. Murai. Effect of wall surface wettability on collective behavior of hydrogen microbubbles rising along a wall. Exp. Therm. Fluid. Sci. 104(15) (2016) 134-140. 